\title{
HEREDITARY CHARACTERISTICS OF THE S339L MUTATION IN A PATIENT WITH MAPLE SYRUP URINE DISEASE IN VIETNAM
}

\author{
Nguyen Thi Thu Huong ${ }^{1,2}$, Vu Chi Dung ${ }^{3}$, Nguyen Thi Thanh Ngan ${ }^{1}$, \\ Nguyen Kim Thoa ${ }^{4}$, Nguyen Huy Hoang,"* \\ ${ }^{1}$ Institute of Genome Research, VAST, Vietnam \\ ${ }^{2}$ Graduate University of Science and Technology, VAST, Vietnam \\ ${ }^{3}$ National Hospital of Pediatrics \\ ${ }^{4}$ Institute of Biotechnology, VAST, Vietnam \\ Received 20 January 2020, accepted 23 April 2020
}

\begin{abstract}
Maple syrup urine disease (MSUD) is an autosomal recessive inherited metabolic disorder caused by malfunction of the branched-chain $\alpha$-ketoacid dehydrogenase complex (BCKDH). This enzyme complex participates in the catalyzing metabolisms of the branched-chain $\alpha$ ketoacids, the second step of the degradation of branched-chain amino acids. Impaired activities of the BCKAD complex lead to an increase of the levels of branched-chain amino acid such as leucine, valine, and isoleucine in the blood. In children with maple syrup urine disease, catalysis of the metabolisms of some amino acids failed to be implemented, leading to an accumulation of the amino acids which has been shown as one of the causes of neurological complications, intellectual disabilities, and nervous paralysis or even death. Pathogenic mutations normally occur in $B C K D H A, B C K D H B, D B T$ and $D L D$ genes which encode the E1 $\alpha$, E1 $\beta$, and E2 subunits of the BCKDH complex. In the present study, a homozygous mutation in the $B C K D H B$ gene (c. $1016 \mathrm{C}>\mathrm{T}$ ) in a pediatric patient with MSUD diagnosed at The National Hospital of Pediatrics was identified using whole exome and Sanger sequencing methods. As a result, the inheritance of the homozygous mutation related to MSUD in $B C K D H B$ gene within the pedigree of the patient's family was determined. The results indicated that the mutation in the $B C K D H B$ gene was inherited from both of the patient's parents. In addition, this finding provides an important scientific basis to researches on MSUD in the Vietnamese population.
\end{abstract}

Keywords: $B C K A D, B C K D H B$, MSUD, S339L, whole exome sequencing.

Citation: Nguyen Thi Thu Huong, Vu Chi Dung, Nguyen Thi Thanh Ngan, Nguyen Kim Thoa, Nguyen Huy Hoang, 2020. Hereditary characteristics of the S339L mutation in a patient with maple syrup urine disease in Vietnam. Academia Journal of Biology, 42(2): 101-107. https://doi.org/10.15625/2615-9023/v42n2.14913.

*Corresponding author email: nhhoang@igr.ac.vn

(C2020 Vietnam Academy of Science and Technology (VAST) 


\section{INTRODUCTION}

Maple syrup urine disease (MSUD), an inherited amino acid metabolism disorder, belongs to a group of rare diseases in newborn babies caused by genetic abnormalities in $B C K D H A, B C K D H B, D B T$ and $D L D$ genes, which encode an inherited amino acid metabolism disorder subunits of the branched $\alpha$-ketoacid dehydrogenase complex (BCKAD). (Lee et al., 2008; Ali \& Ngu, 2018). The BCKDA complex composed of four different subunits E1 $\alpha, \mathrm{E} 1 \beta, \mathrm{E} 2$ and E3 necessary for oxidative decarboxylation of branched-chain $\alpha$-keto acids. The mutational changes of these encoded genes lead to damage functions of the BCKAD complex, resulting in accumulation of branched-chain amino acids, including leucine, isoleucine, and valine. High accumulation of these amino acids could cause mental retardation and neurological impairment. The maple syrup urine disease, if not detected early and treated timely, may lead to seizures, coma, and even death. Based on the genetic mutations, MSUD is classified into four types: type Ia $(B C K D H A)$; type Ib (BCKDHB), type II (DBT) and type III (DLD) (Ali \& Ngu, 2018). Since the first mutation in $B C K D H A$ gene was detected in 1989 (Zhang et al., 1989), more than 283 mutations, scattering over $B C K D H A$, $B C K D H B, D B T$ and $D L D$ genes, been published in the Human Gene Mutation Database (HGMB). Mutations in BCKDHA and $B C K D H B$ genes have been shown to be more common than in $D B T$ and $D L D$ genes. Mutations in MSUD patients can be either by homozygous or heterozygous (Ali \& Ngu, 2018). Based on various clinical presentations, MSUD is classified into five phenotypes, including classic, intermediate, intermittent, thiamine-responsive, and E3deficient phenotypes (Ali \& Ngu, 2018). About $75 \%$ of MSUD patients in classic form are presented at the neonatal stage, presenting normally with neonatal-onset encephalopathy, maple syrup odor in the urine, increased branched chain amino acids in the blood and alpha-ketoacids in the urine (Guo et al., 2015). In this case, the activity of the BCKD enzyme complex is reduced to under $2 \%$ or undetectable (Kerstin et al., 2009; Blackburn et al., 2017). This most dangerous type could be lethal if not detected early and treated promptly. MSUD is classified as a hereditary and very rare disease, appearing in both boys and girls with an estimated incidence rate of 1/185,000 newborn babies (Ali \& Ngu, 2018). However, the number could be much higher in countries with high rates of consanguinity (Kerstin et al., 2009; Jaafar et al., 2013). To date, the incidence rate of MUSD has not been reported in Vietnam. Among congenital metabolic disorders in Vietnam, MUSD is the most common and can be detected at the earliest age. Molecular genetic testing is essential for diagnosis, early detection and treatment of MUSD patients. In the present study, genetic variants and inheritance of the mutations causing MSUD in a pediatric patient and his family members were studied using WES and Sanger.

\section{MATERIALS AND METHODS}

\section{Patient}

Pediatric patient was diagnosed and treated for MSUD at 5 days of age at Department of Medical Genetics, Metabolism and Endocrinology, National Hospital of Pediatrics. Blood samples of the patient and other family members were collected with consent from the patient's parents. This study was performed in accordance with the Declaration of Helsinki, and the protocol approved by the Ethics Committee of the Institute of Genome Research (No.18/QĐ-NCHG).

\section{Clinical presentation}

Clinical information, age of onset, clinical symptoms, results of routine biochemical tests and treatments were collected in the medical records of the Department of Medical Genetics, Metabolism and Endocrinology, National Hospital of Pediatrics.

\section{DNA preparation}

Blood samples were provided by The Department of Medical Genetics, Metabolism 
and Endocrinology, National Hospital of Pediatrics. Total DNA was extracted from whole blood of MSUD patient and his family members using QIAamp DNA Blood Mini Kit (Qiagen, Germany).

\section{Whole exome sequencing and mutation analysis}

The DNA library was prepared using Kit Agilent SureSelect Target Enrichment (Macrogen) following the manufactures' protocol and was sequenced on an Illumina NovaSeq 6000 platform (Illumina, CA, USA). Paired-end sequences were mapped to UCSC/hg19 reference human genome using Burrows-Wheeler Aligner 0.7.12. Duplicates were marked via Picard-1.130. Afterwards, variant data were analyzed using Genome Analysis Toolkit v3.4 and annotated using SnpEff v4.1 with database of dbSNP v142, 1000Genome, ClinVar v 05/2015, ESP. Sift, Polyphen 2 and Mutation Taster were used to evaluate the effect of genetic mutations on protein function. Mutation screening and analysis were performed on $B C K D H A$, $B C K D H B, D B T$ and $D L D$ genes related to MSUD disease.

\section{Sanger sequencing}

An exon 9 region on $B C K D H B$ gene (ENSG 00000083123) was amplified by PCR using specific primers (BCKB-F: 5'TGACCTGTCGAAAGCGAGTT-3', BCKB -R:5'-CTTCTGGAATTGGCATGTGGA-3').

PCR reaction was performed with ingredients of 10X Dream Taq Buffer, $10 \mathrm{mM}$ dNTP, 2.5U/ $\mu 1$ Dream Taq DNA polymerase and 10 pmol $/ \mu 1$ per primer, $100 \mathrm{ng} / \mu \mathrm{l}$ DNA template and thermal cycle: $95^{\circ} \mathrm{C} / 12$ minutes; $\left(95^{\circ} \mathrm{C} / 45\right.$ seconds; $54^{\circ} \mathrm{C} / 45$ seconds; $72^{\circ} \mathrm{C} / 45$ seconds) $\mathrm{x}$ 35 cycles; $72^{\circ} \mathrm{C} / 8 \mathrm{~min}$. The amplified PCR product was checked on $1.0 \%$ agarose gel. PCR product was sequenced using ABI3100
(Applied Biosystems, USA). ClustalX 2 and BioEdit 7.0 were used to analyze the sequencing results to detect genetic mutations by comparing WES sequencing results with reference $B C K D H B$ gene sequence.

\section{RESULTS AND DISCUSSION}

\section{Diagnosis and treatment}

The 5-day-old boy patient is the fifth child of a healthy family with normal parents. His two older sisters died at 27 and 23 days after birth and were diagnosed with MUSD. The patient was admitted at 5 days of age with a short cyanosis occurring alternately. Biochemical test showed high level of leucine (2323 $\mu \mathrm{mol} / \mathrm{l}$, normal: $17-155 \mu \mathrm{mol} / \mathrm{l})$ and allo-isoleucine (74.9 $\mu \mathrm{mol} / 1$, normal: $64-294$ $\mu \mathrm{mol} / \mathrm{l})$. He was managed by stopping feeding, glucose infusion (10 $\mathrm{mg} / \mathrm{kg} / \mathrm{min})$, thiamine supplement and hemofiltration. After 48 hours of treatment, the patient was alert and leucine level was decreased $(432 \mu \mathrm{mol} / \mathrm{l})$. At the age of 23 months, he had 2 recurrent episodes of MUSD and suffered from developmental delay with DQ of $65 \%$. He has been monitored and examined periodically at Department of Medical Genetics, Metabolism and Endocrinology, National Hospital of Pediatrics.

\section{Mutation analysis by WES and Sanger sequencing}

Whole exome sequencing was performed to identify the genetic variants in the patient diagnosed with MSUD. Sequenced results were processed as presented above. WES sequencing exposed a variant on the $B C K D H B$ gene was located on chromosome 6, exon 9, at position 1016 on cDNA and position 339 in the polypeptide sequence (Table 1).

Table 1. Variant c.1016C > T (p.Ser339Leu) on BCKDHB gene was identified by WES in MSUD patients

\begin{tabular}{|c|c|c|c|c|c|c|c|}
\hline Chrom & Gene & $\begin{array}{c}\text { Type of } \\
\text { mutation }\end{array}$ & Zygosity & Exon & $\begin{array}{c}\text { Coding DNA } \\
\text { number }\end{array}$ & $\begin{array}{c}\text { Protein } \\
\text { number }\end{array}$ & dbSNP142_ID \\
\hline chr6 & $B C K D H B$ & Missense & HOM & $9 / 11$ & c.1016C $>\mathrm{T}$ & p.S339L & rs398124561 \\
\hline
\end{tabular}


The Sanger sequencing was used to confirm and analyze the variant in the patient and other family members (father, mother, brother, and sister). BCKB-F and BCKB-R primers were designed to amplify 314 nucleotides on exon 9 of $B C K D H B$ gene. Sanger sequencing showed a homozygous missense mutation c.1016C > $\mathrm{T}$. This mutation occurred in exon 9 , where $\mathrm{C}$ was replaced by $\mathrm{T}$ at position 1016 in
cDNA, resulting in substitution of Ser (Serine) by Leu (Leucine) at position 339 of BCKDHB protein (Figure 1A). Other members of the patient's family (father, mother, brother, and sister) were heterozygous mutation carriers and did not have any clinical presentation. Therefore, the patient with MUSD inherited two copies of the disease causing mutation from both his parents (Figure 1B, 1C).

A
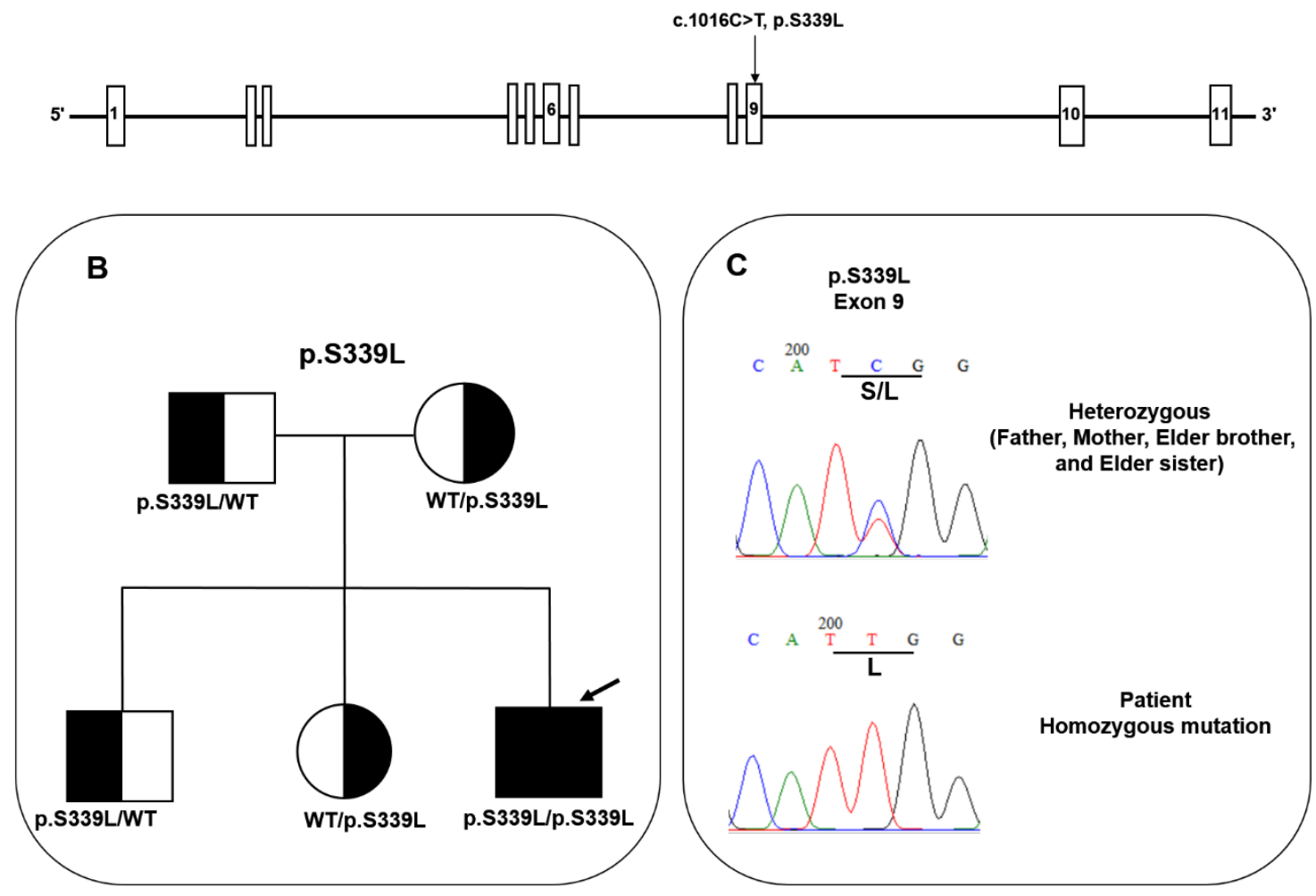

Figure 1. Pedigree and Sanger sequences of pS339L mutation. (A) Location of the mutation on exon 9 of $B C K D H B$ gene. (B) Pedigree presentation of the family of patient. Heterozygous individuals, half black (p.S339L mutation). Patient with homozygous mutation, full black box.

(C) Sanger sequence diagram

The $B C K D H B$ gene encodes one of the four subunits of the BCKAD complex, on the long arm of chromosome 6. Mutations in $B C K D H B$ gene lead to the classic form of MSUD also known as MSUD type Ib (Deepti et al., 2015; Ali \& Ngu, 2018). Previous studies of MSUD indicated that the mutations normally occur in $B C K H D B$ genes (Nellis, Danner, 2001; Skvorak, 2009; Theodoros et al., 2009). Gorzelany K et al. (2009) identified the mutation p.S339L that causes MSUD in Turkish pediatric patient at 18 days of age (Kerstin et al., 2009). This mutation was also found in an one month old Indian girl (Bashyam et al., 2012) and a 6 days old Malaysian girl (Ali \& Ngu, 2018). Thus, S339L on $B C K D H B$ gene was considered as a causative mutation of MSUD. In addition, 
Ser339, located on helix $\alpha 1$ which was linked to Ser339 of E1b subunit via hydrogen bonding, was necessary for the polymerization process between the $\beta$ and $\beta$ subunit (Kerstin et al., 2009; Bashyam et al., 2012). Therefore, the replacement of serine-polar amino acid by leucine non-polar amino acid could disrupt hydrogen bonds, leading to alteration of the $\beta^{\prime} \beta$ arrangement and interaction between these two subunits (Wynn et al. , 2001; Kerstin et al., 2009; Bashyam et al., 2012). Hence, this mutation affected not only the structure, but also the function of BCKDHB protein and was considered to be the cause of MSUD.

\section{Mutation analysis of 3D protein structure model}

PDB software was applied to analyze and predict the effects of genetic variant on the BCKDHB structure. Based on the reference 3D structure from PDB Bank with code
1X7Y, we analyzed the change of amino acids in polypeptide chain when mutations appeared on the BCKHDB protein (Figure 2). In case of no mutation, there were four hydrogen bonds with amino acids Gly336 (two bonds) and Ser343 (two bonds) at Ser339 in the polypeptide chain. When the mutation occurs, the amino acid Ser339 was replaced by Leu339 at Leu339, resulting in a loss of hydrogen bond with the amino acid Gly336 (Figure 2). This change affected the structure as well as the function of BCKHDB protein.

In addition, Bashyam et al. (2012) used PyMOL to predict the effect of p.S339L mutation on the structure of BCKHDB protein. The result indicated that replacing a polar amino acid serine by a hydrophobic amino acid leucine lead to a break the hydrogen bond between two subunits of E1, resulting in a change of BCKHDB secondary structure (Bashyam et al., 2012).
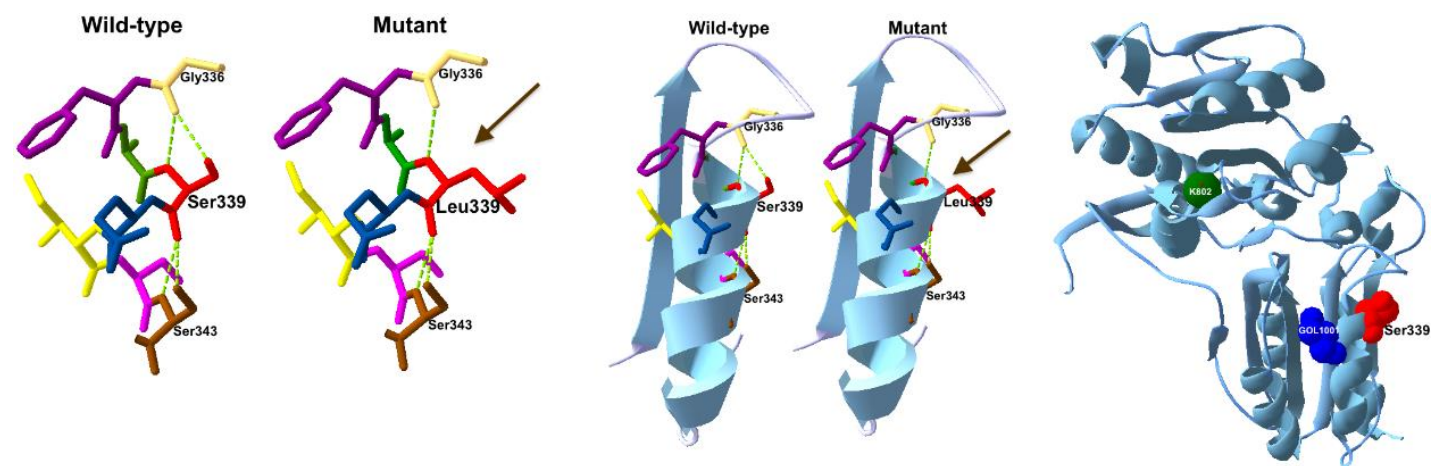

Figure 2. Model of BCKDHB 3D structure containing p.S339L mutation in BCKDHB protein. Leu339 was marked by the brown arrow

\begin{tabular}{|c|c|c|}
\hline Species & $\begin{array}{c}\text { Codon } \\
\text { number }\end{array}$ & Amino acid sequence \\
\hline Homo sapients & 220 & EAPLTGGFASEISSTVQEECFLNLEA \\
\hline Pan paniscus & 220 & EAPLTGGFASEISSTVQEECFLNLEA \\
\hline Nomascus leucogenys & 219 & EAPLTGGFASEISSTVQEECFLNLEA \\
\hline Pongo abelii & 220 & EAPLTGGFASEISSTVQEECFLNLEA \\
\hline Delphinapterus leucas & 223 & EAPLTGGFASEISSTVQEECFLNLEA \\
\hline Monodon monoceros & 223 & EAPLTGGFASEISSTVQEECFLNLEA \\
\hline Marmota marmota marmota & 220 & EAPLTGGFASEISSTVQEECFLNLEA \\
\hline Castor fiber & 221 & EAPLTGGFASEISSTVQEECFLNLEA \\
\hline Oryctolagus cuniculus & 220 & EAPLTGGFASEISSTVQEECFLNLEA \\
\hline Castor canadensis & 221 & EAPLTGGFASEISSTVQEECFLNLEA \\
\hline
\end{tabular}

Figure 3. Amino acid sequence of protein of several species in comparison with human species 
Moreover, multiple alignment of amino acid sequences of BCKDHB against mutation positions across species, including human (Homo sapiens), bonobo (Pan paniscus), white-cheeked gibbon (Nomascus leucogenys), sumatran orangutans (Pongo abelii), white whales (Delphinapterus leucas), narwhal (Monodon monoceros), marmota marmota (Marmota marmota marmota), Eurasian beaver (Castor fiber), European rabbit (Oryctolagus cuniculus), American beaver (Castor canadensis) was performed using ClustalX 2. Conservation analysis indicated that p.S339L mutation occurrs at highly evolutionary conserved position (Figure 3).

\section{CONCLUSION}

In the present study, we reported a homozygous missense mutation c. $1016 \mathrm{C}>\mathrm{T}$ (p.S339L) in $B C K D H B$ gene, which was inherited from both parents in a boy patient with MSUD in Vietnam. His father, mother, brother, and sister did not show any clinical presentation due to the heterozygous mutation. The obtained result accurately determined the genetic cause of disease in the patient's family. In addition, this study is the basis for genetic counseling through newborn screening for diagnosis, early detection and treatment.

Acknowledgments: This work was financially supported by Vietnam Academy of Science and Technology (grant number: KHCBSS.02/18-20).

\section{REFERENCES}

Ali E. Z., Ngu L. H., 2018. Fourteen new mutations of $B C K D H A, B C K D H B$ and $D B T$ genes associated with maple syrup urine disease (MSUD) in Malaysian population. Mol. Genet. Metab. Ref., 17: 22-30.

Bashyam M. D., Chaudhary A. K., Sinha M., Nagarajaram H. A., Devi A. R. R., Bashyam L., Reddy E., Dalal A., 2012. Molecular genetic analysis of MSUD from India reveals mutations causing altered protein truncation affecting the $\mathrm{C}$-termini

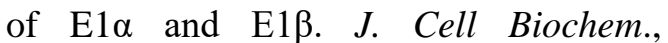
113(10): 3122-3132.

Blackburn P. R., Gass J. M., Pinto E., Vairo F., Farnham K. M., Atwal H. K., Macklin S., Klee E., Atwal P. S., 2017. Maple syrup urine disease: mechanisms and management. Appl. Clin. Genet., 10: $57-66$.

Deepti G., Sunita B. M., Renu S., Sudha K., Ratna D. P., Jyotsna V., Thomas E., Yosuke S., Seiji Y., Roumi D., Ishwar C. V., 2015. Identification of mutations, genotype-phenotype correlation and prenatal diagnosis of maple syrup urine disease in Indian patients. Eur. J. Med. Genet., 58: 471-478.

Guo Y., Liming L., Jiang L., 2015. Two novel compound heterozygous mutations in the $B C K D H B$ gene that cause the intermittent form of maple syrup urine disease. Metab. Brain Dis., 30(6): 1395-1400.

Jaafar N., Moleirinho A., Kerkeni E., Monastiri K., Seboui H., Amorim A., Prata M., Quental S., 2013. Molecular characterization of maple syrup urine disease patients from Tunisia. Gene, 517(1): 116-119.

Kerstin G., Ali D., Turgay C., Serap H. K. S., Gülden F. G., Mübeccel D., Oliver F., Udo W., 2009. Molecular genetics of maple syrup urine disease in the Turkish population. Turk. J. Pediatr., 51: 97-102.

Lee J. Y., Chiong M. A., Estrada S. C., Cutiongco-De La Paz E. M., Silao C. L. T., Padilla C. D., 2008. Maple syrup urine disease (MSUD) - Clinical profile of 47 Filipino patients. J. Inherit. Metab. Dis., 31 (Suppl.2).

Nellis M. M., Danner D. J., 2001. Gene preference in maple syrup urine disease. Am. J. Hum. Genet., 68: 232-237.

Skvorak K. J., 2009. Animal models of maple syrup urine disease. J. Inherit. Metab. Dis., 229-246.

Theodoros G., Jacinta L. C., Max W. R., Goula S., Mark K., David T. C., Anthi D., 2009. Maple syrup urine disease in 
Cypriot families: identification of three novel mutations and biochemical characterization of the p.Thr211Met mutation in the E1alpha subunit. Genet. Test Mol. Biomark., 13: 657-664.

Wynn R. M., Chuang J. L., Sansaricq C., Mandel H., Chuang D. T., 2001. Biochemical basis of type Ib (E1 $\beta)$ mutations in maple syrup urine disease. A prevalent allele in patients from the druze kindred in Israel. J. Biol. Chem., 276(39): 36550-36556.

Zhang B., Kuntz M. J., Goodwin G. W., Edenberg H. J., Crabb D. W., Harris., Ramzan H. K., 1989. cDNA cloning of the E1 alpha subunit of the branched chain alpha-keto acid dehydrogenase and elucidation of a molecular basis for maple syrup urine disease. Ann. NY Acad. Sci., 573: 130-136. 